\title{
An Eulerian interface-sharpening algorithm for compressible gas dynamics *
}

\author{
Keh-Ming Shyue \\ Department of Mathematics, National Taiwan University, Taipei 10617, Taiwan \\ shyue@ntu.edu.tw
}

Summary. We describe a novel Eulerian interface-sharpening approach for the efficient numerical resolution of contact discontinuities arising from inviscid compressible flow in more than one space dimension. The algorithm uses the single-phase compressible Euler equations as the model system, and introduces auxiliary differential terms to the model so as to neutralize numerical diffusion that is inevitable when the original Euler system is solved by a diffused interface method. A standard fractional-step method is employed to solve the proposed model equations in two steps, yielding an easy implementation of the algorithm. Preliminary results obtained using an anti-diffusion based model system are shown to demonstrate the feasibility of the algorithm for practical problems.

\section{Introduction}

Computing a non-oscillatory, positivity-preserving, sharply resolved volume fraction function, denoted by $\alpha \in[0,1]$, for the initial-value problem of the volume-fraction transport equation

$$
\partial_{t} \alpha+\mathbf{u} \cdot \nabla \alpha=0
$$

with discontinuous initial data is of fundamental importance in many practical problems of interest. One simple example is concerned with an unsteady, incompressible, viscous, two-phase flow that is governed by the incompressible Navier-Stokes equations,

$$
\begin{aligned}
\nabla \cdot \mathbf{u} & =0 \\
\partial_{t}(\rho \mathbf{u})+\nabla \cdot\left(\rho \mathbf{u} \otimes \mathbf{u}+p I_{N}\right) & =\nabla \cdot \boldsymbol{\tau}+\rho \mathbf{g}+\mathbf{f}_{\sigma},
\end{aligned}
$$

where $\mathbf{u}$ denotes the velocity vector, $\rho$ the density, $p$ the pressure, $I_{N}$ the $N \times N$ identity matrix ( $N$ the number of spatial dimensions), $\boldsymbol{\tau}=\epsilon\left(\nabla \mathbf{u}+\nabla \mathbf{u}^{T}\right)$ the stress tensor, $\mathbf{g}$ the gravitational field, and $\mathbf{f}_{\sigma}=-\sigma \kappa \nabla \alpha$ the capillary force. We assume that the fluids of interest consist of two different phases, gas and liquid, for instance, separated by immiscible interfaces, where in regions $\alpha=0$ and $\alpha=1$, the fluid is

\footnotetext{
* This work was supported in part by National Science Council of Taiwan Grants \#96-2115-M-002-008-MY3 and 99-2115-M-002-005-MY2.
} 
single phase (gas or liquid), while in regions $0<\alpha<1$, we have a (gas-liquid) two-phase coexistent phase. In the latter case, it is a common practice to set the density as well as the material quantities such as the dynamic viscosity $\epsilon$ and the surface-tension coefficient $\sigma$ by the solution of (1) via a simple $\alpha$-weighted average,

$$
z=\alpha z_{1}+(1-\alpha) z_{2}
$$

for $z=\rho, \epsilon, \sigma ; z_{k}$ the $k$ th phasic variable of $z$. In addition to that, from the given set of volume fractions, the normal direction $\nabla \alpha$ and the curvature $\kappa=\nabla \cdot(\nabla \alpha /|\nabla \alpha|)$ at the interface that contributes to the capillary force $\mathbf{f}_{\sigma}$ on the right-hand side of the momentum equations may be calculated via numerical means.

For incompressible two-phase flow governed by (1) and (2), interface sharpening of some kind (cf. $[1,8,15,16,17]$ and references therein) is a popular technique that is applied together with an underlying advection scheme to compute a sharp solution profile of (1); this yields an accurate definition of the aforementioned physical and geometrical quantities present in (2) near the interfaces, and is viable to the remaining parts of the flow solver. Among various interface-sharpening approaches, in this work, we are interested in a class of methods that is based on the inclusion of a differential source term to (1) in a form,

$$
\partial_{t} \alpha+\mathbf{u} \cdot \nabla \alpha=\frac{1}{\mu} \mathcal{D}_{\alpha}
$$

as a numerical model for interface-sharpening, where $\mu \in \mathbb{R}$ is a free parameter. In the work proposed by Olsson, Kreiss, and coworker (cf. $[9,10])$, the term $\mathcal{D}_{\alpha}$ is of an interface-compression type as

$$
\mathcal{D}_{\alpha}:=\nabla \cdot[(\varepsilon \nabla \alpha \cdot \mathbf{n}-\alpha(1-\alpha)) \mathbf{n}],
$$

where both a nonlinear convection and a linear diffusion term are introduced in the model. Here $\mathbf{n}=\nabla \alpha /|\nabla \alpha|$ is the unit normal, and $\varepsilon>0$ is the diffusion coefficient which is assumed to be in the order of the spatial mesh size. On the other hand, in the work advocated by So, $\mathrm{Hu}$, and Adams [13], it takes simply the linear diffusion term, but is of an anti-diffusion one, as

$$
\mathcal{D}_{\alpha}:=-\nabla \cdot(\varepsilon \nabla \alpha),
$$

where the diffusion coefficient $\varepsilon$ is assumed to be in the order of the velocity vector in absolute value which mimics the diffusion rate from the modified equation of the numerical method.

Our goal here is to describe a novel approach that generalizes (3) for interfacesharpening of discontinuous volume fractions in incompressible flow to more general interfaces (i.e., contact discontinuities) that are governed by the Euler equations in the compressible single-phase flow; an extension of this approach to the multiphase case will be reported elsewhere. The proposed model that we are going to describe in Section 2 will be formulated in such a way that a standard fractional-step method can be applied, yielding a simple and yet accurate algorithm for numerical approximation. 


\section{Mathematical Models}

The basic physical conservation laws for the inviscid, non-heat conducting, singlephase, compressible flow in Cartesian coordinates take the form

$$
\begin{aligned}
\partial_{t} \rho+\nabla \cdot(\rho \mathbf{u}) & =0, \\
\partial_{t}(\rho \mathbf{u})+\nabla \cdot\left(\rho \mathbf{u} \otimes \mathbf{u}+p I_{N}\right) & =0, \\
\partial_{t} E+\nabla \cdot(E \mathbf{u}+p \mathbf{u}) & =0 .
\end{aligned}
$$

We assume that the constitutive law for the fluid phase of interest satisfies a MieGrüneisen equation of state of the form

$$
p(\rho, e)=p_{\text {ref }}(\rho)+\Gamma(\rho) \rho\left[e-e_{\text {ref }}(\rho)\right] .
$$

Here $e$ denotes the specific internal energy, $\Gamma=\left.(1 / \rho)\left(\partial_{e} p\right)\right|_{\rho}$ is the Grüneisen coefficient, and $p_{\text {ref }}, e_{\text {ref }}$ are the properly chosen states of the pressure and the internal energy along some reference curve (e.g., along an isentrope, a single shock Hugoniot, or the other empirically fitting curves) in order to match the experimental data of the material being examined. For simplicity, each of the expressions $\Gamma, p_{\text {ref }}$, and $e_{\text {ref }}$ is taken as a function of the density only, see Section 4 for an example. We have $E=\rho e+\rho \mathbf{u} \cdot \mathbf{u} / 2$ denoting the total energy as usual.

To derive our compressible model for interface-sharpening that may be used in a diffused interface method for numerical approximation, as in our previous work for compressible multiphase flow solver (cf. [12]), we begin by considering an interface only problem (i.e., a contact discontinuity in gas dynamics) where both the pressure and the velocity are assumed to be constants in the whole domain, while the density is having jumps across some interfaces. Then from (4), we find easily the basic transport equations for the interfaces as

$$
\begin{aligned}
\partial_{t} \rho+\mathbf{u} \cdot \nabla \rho & =0, \\
\mathbf{u}\left(\partial_{t} \rho+\mathbf{u} \cdot \nabla \rho\right) & =0, \\
\frac{\mathbf{u} \cdot \mathbf{u}}{2}\left(\partial_{t} \rho+\mathbf{u} \cdot \nabla \rho\right)+\left[\partial_{t}(\rho e)+\mathbf{u} \cdot \nabla(\rho e)\right] & =0 .
\end{aligned}
$$

With the interface-sharpening model (3) for the volume fraction in mind, it should be sensible to assume a variant model for the density as

$$
\partial_{t} \rho+\mathbf{u} \cdot \nabla \rho=\frac{1}{\mu} \mathcal{D}_{\rho},
$$

where the term $\mathcal{D}_{\rho}$ can be defined analogously based on either the interfacecompression or the anti-diffusion formulation. Having that, to ensure the velocity remains at a constant state across the interfaces Eq. (6b) should be modified by

$$
\mathbf{u}\left(\partial_{t} \rho+\mathbf{u} \cdot \nabla \rho\right)=\frac{1}{\mu} \mathbf{u} \mathcal{D}_{\rho} .
$$

Furthermore, to ensure the pressure retains in equilibrium also, using the equation of state (5) and Eq. (6a), together with a proper smoothness assumption of the density, it is not difficult to show that Eq. (6c) should be modified by

$$
\frac{\mathbf{u} \cdot \mathbf{u}}{2}\left(\partial_{t} \rho+\mathbf{u} \cdot \nabla \rho\right)+\left[\partial_{t}(\rho e)+\mathbf{u} \cdot \nabla(\rho e)\right]=\frac{1}{\mu}\left[\frac{\mathbf{u} \cdot \mathbf{u}}{2}+\partial_{\rho}(\rho e)\right] \mathcal{D}_{\rho},
$$


where we have $\partial(\rho e) / \partial \rho=e_{\text {ref }}+\rho e_{\text {ref }}^{\prime}-\left(\Gamma p_{\text {ref }}^{\prime}+\left(p-p_{\text {ref }}\right) \Gamma^{\prime}\right) / \Gamma^{2} ; z^{\prime}=d z / d \rho$ for $z=\Gamma, p_{\text {ref }}$, and $e_{\text {ref }}$.

Since in general we are interested in shock wave problems as well, we should apply the interface-sharpening terms described above only locally near the interfaces. For this reason, it is common to introduce an interface indicator, denoted by $H_{I}$, to the model so that it takes effect near the interfaces only, and has no effect on the other genuinely nonlinear shock and rarefaction waves (cf. [11]).

With that, in summary, the interface-sharpening model we propose to solve inviscid compressible single-phase flows with the Mie-Grüneisen equation of state (5) in Cartesian coordinates takes the form

$$
\begin{aligned}
\partial_{t} \rho+\nabla \cdot(\rho \mathbf{u}) & =\frac{1}{\mu} H_{I} \mathcal{D}_{\rho}, \\
\partial_{t}(\rho \mathbf{u})+\nabla \cdot\left(\rho \mathbf{u} \otimes \mathbf{u}+p I_{N}\right) & =\frac{1}{\mu} H_{I} \mathcal{D}_{\rho \mathbf{u}} \\
\partial_{t} E+\nabla \cdot(E \mathbf{u}+p \mathbf{u}) & =\frac{1}{\mu} H_{I} \mathcal{D}_{E}
\end{aligned}
$$

Here, without causing any confusion, in Eq. (8) we have used the notations $\mathcal{D}_{\rho \mathbf{u}}:=$ $\mathbf{u} \mathcal{D}_{\rho}$, and $\mathcal{D}_{E}:=(\mathbf{u} \cdot \mathbf{u} / 2-\partial(\rho e) / \partial \rho) \mathcal{D}_{\rho}$.

To end this section, for the ease of the latter discussion, it is useful to write (8) into a dimension-wise expression by

$$
\partial_{t} q+\sum_{j=1}^{N} \partial_{x_{j}} f_{j}(q)=\frac{1}{\mu} \psi(q),
$$

with $q, f_{j}$, and $\psi$ defined respectively by

$$
\begin{aligned}
q & =\left(\rho, \rho u_{1}, \ldots, \rho u_{N}, E\right)^{T} \\
f_{j} & =\left(\rho u_{j}, \rho u_{1} u_{j}+p \delta_{j 1}, \ldots, \rho u_{N} u_{j}+p \delta_{j N}, E u_{j}+p u_{j}\right)^{T}, \\
\psi & =H_{I}\left(\mathcal{D}_{\rho}, \mathcal{D}_{\rho u_{1}}, \ldots, \mathcal{D}_{\rho u_{N}}, \mathcal{D}_{E}\right)^{T}
\end{aligned}
$$

where $\delta_{i j}$ is the Kronecker delta.

\section{Numerical Methods}

To approximate (9) numerically, a fractional step method that consists of the following steps in each time iteration is employed:

(1) Solve the model equation without interface-sharpening terms

$$
\partial_{t} q+\sum_{j=1}^{N} \partial_{x_{j}} f_{j}(q)=0
$$

using a state-of-the-art shock-capturing method over a time step $\Delta t$. 
(2) Iterate the equation with the interface-sharpening terms

$$
\partial_{\tau} q=\psi(q)
$$

using a simple explicit method, over a time step $\Delta \tau$ towards a "sharp layer"; $\tau=t / \mu$ is a scaled time variable.

Note that in step 1 we have used a standard high-resolution finite-volume method based on a wave-propagation viewpoint for the numerical approximation of Eq. (10a) (cf. [5]), and in step 2 we have employed an explicit first-order in time and secondorder in space finite difference method for discretizing Eq. (10b) (cf. [6, 11, 14]). In this work, the local interface indicator $H_{I}$ is defined as a Heaviside function of form

$$
H_{I}(z)= \begin{cases}1 & \text { if } z \geq z_{0} \\ 0 & \text { otherwise }\end{cases}
$$

where the variable $z$ can be taken as some measure of the physical quantities such as density, entropy, pressure, and velocity. Alternatively, it can be taken on an augmented variable such as the volume fraction that is introduced to Eq. (8) for that matter. Here $z_{0}$ is some prescribed tolerance on $z$, see [11] for the other approach.

In the numerical results shown in Section 4, an anti-diffusion based model equation with $\mathcal{D}_{\rho}=-\nabla \cdot(\varepsilon \nabla \rho)$ is used in the model for approximation. Here the diffusion coefficient $\varepsilon$ is chosen to be a function of the local velocity that varies both in space and time. To stabilize the computation of $\nabla \rho$ and so the flux $\varepsilon \nabla \rho$, the MinmoD limiter is imposed in step 2 of the method (cf. $[2,3]$ ). As to the stopping criterion towards a "sharp layer", in practice, only 1 or 2 iterations are sufficient for the interface-sharpening purpose.

\section{Numerical Examples}

We now present sample results obtained using our interface-sharpening method with anti-diffusion based model equations in both one and two dimensions. Additional results that further validate the proposed method will be reported elsewhere.

EXAMPLE 4.1. Our first test problem is the classical Sod shock tube problem in one dimension, where initially the state variables are $\left(\rho, u_{1}, p\right)_{L}=(1,0,1)$ and $\left(\rho, u_{1}, p\right)_{R}=(0.125,0,1)$, respectively. Here $L$ is the state used for $x_{1} \in[0,0.5)$, and $R$ is the state used for $x_{1} \in[0.5,1]$. The fluid inside the domain is gas modeled by the ideal gas equation of state $p(\rho, e)=(\gamma-1) \rho e$ with $\gamma=1.4$. There are non-reflecting outflow boundaries on the left and right sides.

In Fig. 1, we show interface-sharpening results for the density, velocity, and pressure at time $t=0.15$ using a 100 grid. It is easy to see that our interface-sharpening algorithm works in a satisfactory matter on the interface without introducing any spurious oscillations in the pressure, while retaining the same solution structure in the region of shock and rarefaction waves.

EXAMPLE 4.2. Our second example in one dimension is an impact problem in which a pre-compressed semi-infinite aluminum slab at rest with $(\rho, p)=$ $\left(4000 \mathrm{~kg} / \mathrm{m}^{3}, 7.93 \times 10^{9} \mathrm{~Pa}\right)$ is being hit by an ambient aluminum slab traveling at the speed $2 \mathrm{~km} / \mathrm{s}$ from the right to the left with the reference state $(\rho, p)=\left(\rho_{0}, p_{0}\right)$. We assume that the constitutive law of an aluminum satisfies the Mie-Grüneisen equation of state (5) with $\Gamma, p_{\text {ref }}$, and $e_{\text {ref }}$ defined by 

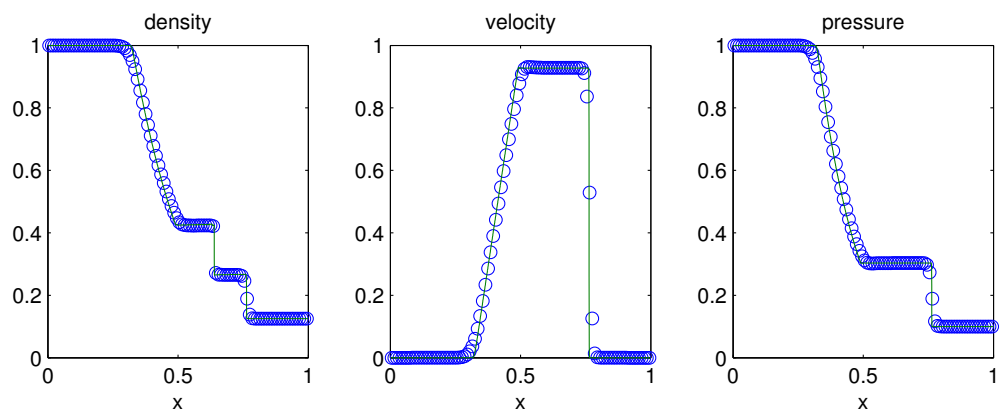

Fig. 1. Interface-sharpening result for the Sod shock tube problem at time $t=0.15$ using a 100 grid. The solid line shown in the graph is the exact solution.

$$
\Gamma(\rho)=\Gamma_{0}(1-\eta)^{\alpha}, \quad p_{\text {ref }}(\rho)=\frac{\rho_{0} c_{0}^{2} \eta}{(1-s \eta)^{2}}, \quad e_{\text {ref }}(\rho)=\frac{\eta}{2 \rho_{0}}\left(p_{0}+p_{\text {ref }}(\rho)\right),
$$

where the numerical values of the material constants are taken to be $\rho_{0}=2785 \mathrm{~kg} / \mathrm{m}^{3}$, $p_{0}=0, c_{0}=5328 \mathrm{~m} / \mathrm{s}, s=1.338, \Gamma_{0}=2$, and $\alpha=1 ; \eta=1-\rho_{0} / \rho$.

In this setup, it is not difficult to show that the exact solution of this problem would consist of a leftward going shock wave to the stationary aluminum, a material interface, and a rightward going shock wave to the moving aluminum. Figure 2 shows results with and without interface-sharpening at time $t=50 \mu \mathrm{s}$ using a 200 grid. From the figure, we observe a slight improvement on the interface structure, see [12] also for a similar calculation.
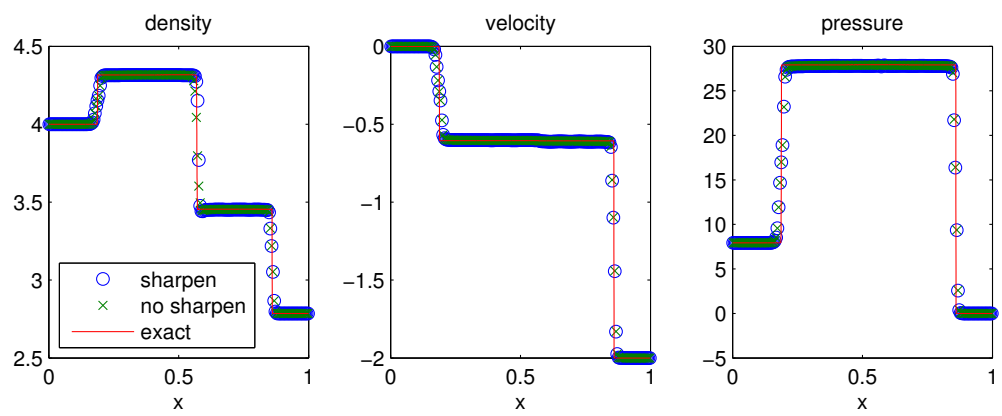

Fig. 2. Interface-sharpening result for the aluminum impact problem at time $t=$ $50 \mu$ s using 200 grids. The solid line shown in the graph is the exact solution and the symbol " $\times$ " is the result obtained using the method without interface-sharpening.

EXAMPLE 4.3. We are next concerned with a passive evolution of a twodimensional square column of size $\left(x_{1}, x_{2}\right) \in[0.3,0.7] \times[0.3,0.7] \mathrm{m}^{2}$ in a unit square domain with uniform equilibrium pressure $p=10^{5} \mathrm{~Pa}$ and constant particle velocity $\left(u_{1}, u_{2}\right)=\left(10^{2} \mathrm{~m} / \mathrm{s}, 10^{2} \mathrm{~m} / \mathrm{s}\right)$. In this test, the density in the region inside a square column is $1500 \mathrm{~kg} / \mathrm{m}^{3}$, and it is $1000 \mathrm{~kg} / \mathrm{m}^{3}$ otherwise. We use the linearized MieGrüneisen equation of state $p(\rho, e)=(\gamma-1) \rho e+c_{0}^{2}\left(\rho-\rho_{0}\right)$ to model the material 
in the whole domain with the material-dependent quantities taken to be $\gamma=4.4$, $\rho_{0}=1000 \mathrm{~kg} / \mathrm{m}^{3}, c_{0}=1624 \mathrm{~m} / \mathrm{s}$. Figure 3 shows contour plots of the density obtained using the method with and without interface-sharpening at time $t=0.02 \mathrm{~s}$ using a $100 \times 100$ grid. An excellent interface-sharpening result is observed, whereas a severely diffused interface is seen using the standard high-resolution method. Here periodic boundary conditions are used on all sides.

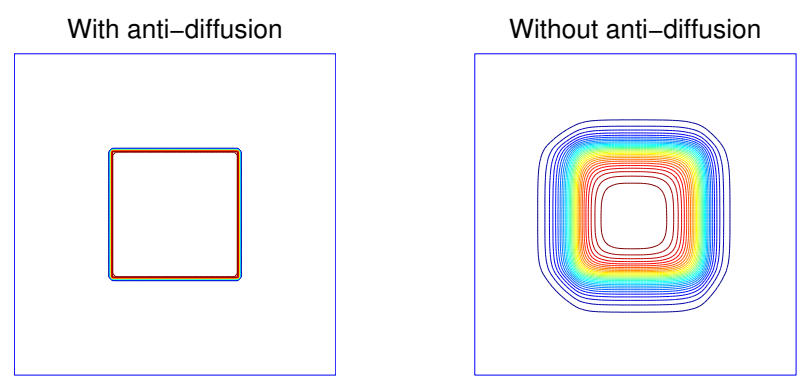

Fig. 3. Numerical results for a passive evolution of a square column. Contours of the density are shown at time $t=0.02 \mathrm{~s}$ obtained using the method with and without interface-sharpening on a $100 \times 100$ grid.

EXAMPLE 4.4. Our second example in two dimensions is a Mach 3 shock wave in air interacting with a heavier circular gas column. In this test, we take a shock tube of size $\left(x_{1}, x_{2}\right) \in[-1,1] \times[0,0.5] \mathrm{m}^{2}$, and consider a planarly leftward-moving shock wave with initial position $x_{1}=0.7 \mathrm{~m}$ and states in the pre- and post-shock as

$$
\begin{aligned}
\left(\rho, u_{1}, u_{2}, p\right)_{\text {pre-shock }} & =\left(1 \mathrm{~kg} / \mathrm{m}^{3}, 0,0,10^{5} \mathrm{~Pa}\right), \\
\left(\rho, u_{1}, u_{2}, p\right)_{\text {post-shock }} & =\left(3.857 \mathrm{~kg} / \mathrm{m}^{3},-831.479 \mathrm{~m} / \mathrm{s}, 0,1.033 \times 10^{6} \mathrm{~Pa}\right),
\end{aligned}
$$

respectively. In addition to that, we assume a stationary heavier circular gas column with radius $0.2 \mathrm{~m}$ and center $(0.4,0) \mathrm{m}$ lying in front of the shock. Inside the gas column the flow is in standard atmospheric condition with density $\rho=10 \mathrm{~kg} / \mathrm{m}^{3}$; this gives us one example that the interface is accelerated by a shock wave coming from the light-fluid to the heavy-fluid region, yielding a transmitted shock wave, an interface, and a reflected shock after the interaction. As in EXAMPLE 4.1, the fluid under consideration is an ideal gas with $\gamma=1.4$.

In Fig. 4, we show schlieren images of density obtained using the method with and without interface-sharpening at six different times $t=2^{i} \times 10^{-1} \mathrm{~ms}$ for $i=$ $1,2, \ldots, 6$, with a $800 \times 200$ grid. It is interesting to see that as far as the global wave structure (i.e., the shape and location of the incident, transmitted and reflected waves) is concerned, we observe similar behavior of the solutions between those two. However, a sharper resolution of the contact line is observed when our anti-diffusion method is in use.

EXAMPLE 4.5. Finally, we are interested in a model blast wave problem with complex wave interactions and a general equation of state. As initial condition, we have a stationary circular gaseous explosive charge of radius $0.1 \mathrm{~m}$ and center $(0,0.25) \mathrm{m}$ located in a rectangular domain $\left(x_{1}, x_{2}\right) \in[-1,1] \times[0,1] \mathrm{m}^{2}$. Inside the 

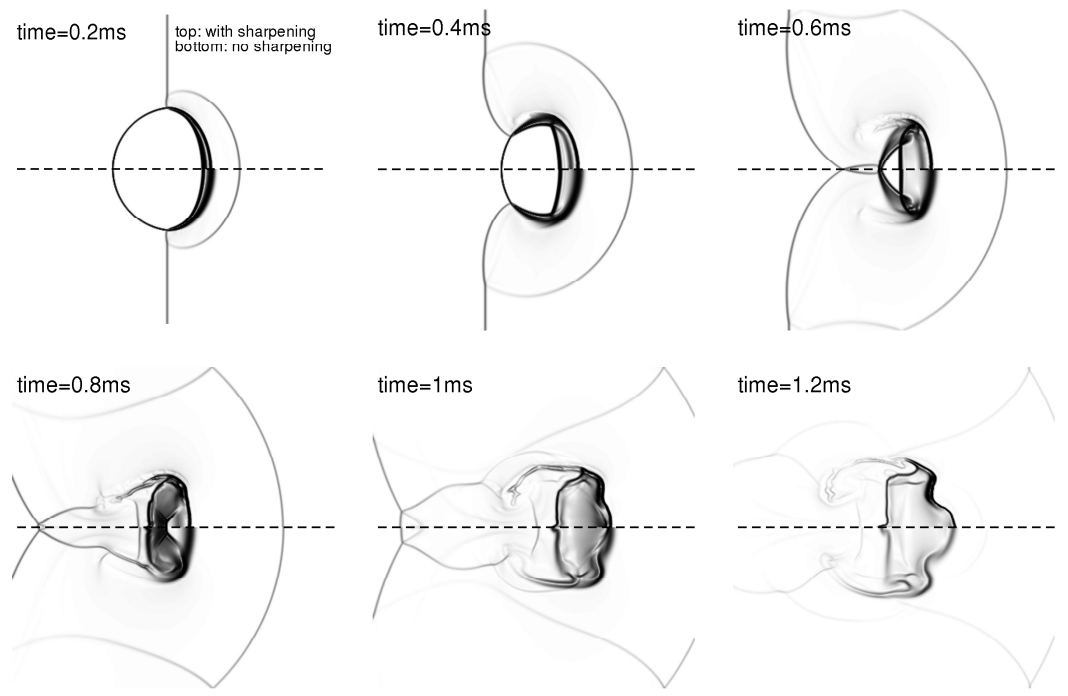

time $=1.2 \mathrm{~ms}$

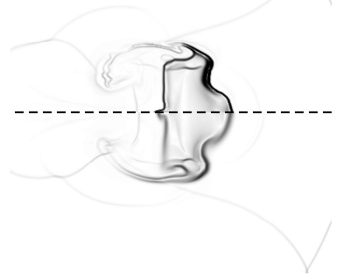

Fig. 4. Numerical results for a Mach 3 shock wave in air interacting with a heavier circular gas column. Schlieren images of density are shown at six different times $t=2^{i} \times 10^{-1} \mathrm{~ms}$ for $i=1,2, \ldots, 6$, obtained using the method with and without interface-sharpening (drawn on the top and bottom parts of each graph, respectively) with a $800 \times 200$ grid.

circular region, the density and pressure are $\rho=1700 \mathrm{~kg} / \mathrm{m}^{3}$ and $p=10^{12} \mathrm{~Pa}$, while outside the circular region, we have $\rho=1000 \mathrm{~kg} / \mathrm{m}^{3}$ and $p=5 \times 10^{10} \mathrm{~Pa}$. The material in the entire domain is modeled by the Jones-Wilkins-Lee equation of state for gaseous explosives (cf. [4]) in that it takes the form (5) with

$$
\begin{aligned}
\Gamma(V) & =\Gamma_{0}, \quad p_{\mathrm{ref}}(V)=\mathcal{A} \exp \left(\frac{-\mathcal{R}_{1} V}{V_{0}}\right)+\mathcal{B} \exp \left(\frac{-\mathcal{R}_{2} V}{V_{0}}\right), \\
e_{\mathrm{ref}}(V) & =\frac{\mathcal{A} V_{0}}{\mathcal{R}_{1}} \exp \left(\frac{-\mathcal{R}_{1} V}{V_{0}}\right)+\frac{\mathcal{B} V_{0}}{\mathcal{R}_{2}} \exp \left(\frac{-\mathcal{R}_{2} V}{V_{0}}\right)-e_{0},
\end{aligned}
$$

where $V=1 / \rho$ is the specfic volume. The material-dependent quantities we use in the simulations are $\Gamma_{0}=0.28, \rho_{0}=1640 \mathrm{~kg} / \mathrm{m}^{3}, e_{0}=0, \mathcal{A}=494 \mathrm{GPa}, \mathcal{B}=1.21 \mathrm{GPa}$, $\mathcal{R}_{1}=4.94$, and $\mathcal{R}_{2}=1.21$. The boundary conditions are solid walls on the top- and bottom-side, and non-reflecting on the left- and right-side.

In this problem, due to the pressure difference, breaking of the circular membrane occurs instantaneously, yielding an outward-going shock wave, an inward-going rarefaction wave, and a contact discontinuity lying in between. At a later time, this outward-going shock wave is reflected from the bottom wall, and so the inward-going rarefaction is bounced back from the explosive center; this generates complex wave interactions afterwards.

Figure 5 shows schlieren images of density at three different times $t=18,36$, and $72 \mu \mathrm{s}$. Here we have performed the computations using both the anti-diffusion based interface-sharpening method with a $400 \times 200$ grid, and also the local adaptive 
mesh refinement (AMR) version of the method without anti-diffusion (cf. [7]). In the later AMR runs, the base grid used here is $200 \times 100$ and with a two-level of grid refinement; the refinement ratio is 4 for each level of grid, i.e., in the refined region the mesh size is twice smaller than in the anti-diffusion runs. From the figure, we observe the same qualitative structure of solution between them, especially on the structure of shock waves; this is as expected because for the Euler equations without any source terms the shock wave is stable under mesh refinement. This is not the case, however, for interfaces (contact discontinuities); a difference in the solution then occurs due to the perturbation of complex wave interactions upon them.

With interface-sharpening

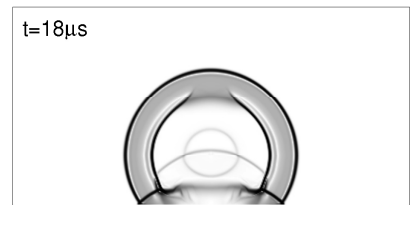

$\mathrm{t}=36 \mu \mathrm{s}$
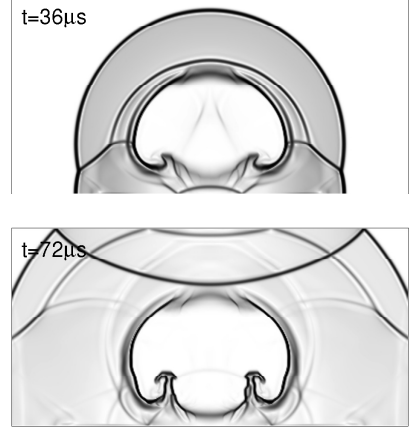

With AMR
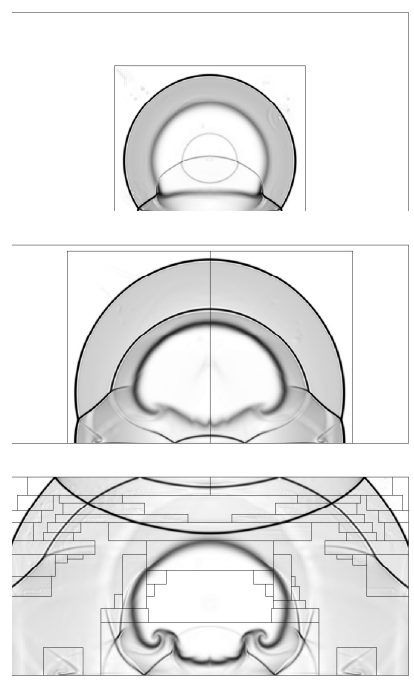

Fig. 5. Numerical results for a blast wave problem in two dimensions. Schlieren images of density are shown at three different times $t=18,36,72 \mu$ s obtained using the methods with anti-diffusion (the left column) and with local adaptive mesh refinement (the right column).

\section{Conclusion}

We have described a class of interface-sharpening methods for single-phase compressible flow with interfaces. Numerical validation of the proposed methods using an anti-diffusion based model system has been performed. It shows the feasibility of the algorithm for sharpening compressible interfaces numerically in one and two dimensions. Ongoing work is to validate the method using the interface-compression based model, and extend the method to compressible multiphase flow and to mapped grids with complex geometries. 


\section{References}

1. J. P. Boris and D. L. Book. Flux-corrected transport I. SHASTA, a fluid transport algorithm that works. J. Comput. Phys., 11:38-69, 1973.

2. M. Breuß, T. Brox, T. Sonar, and J. Weickert. Stabilized nonlinear inverse diffusion for approximating hyperbolic PDEs. In R. Kimmel, N. Sochen, and J. Weickert, editors, Proceedings Scale Space 2005, Springer LNCS 3459, pages 536-547. Springer, 2005.

3. M. Breuß and M. Welk. Staircasing in semidiscrete stabilized inverse linear diffusion algorithms. J. Comput. Appl. Math., 206:520-533, 2007.

4. B. M. Dobratz and P. C. Crawford. LLNL Explosive handbook: properties of chemical explosives and explosive simulants. UCRL-52997, LLNL, 1985.

5. R. J. LeVeque. Finite Volume Methods for Hyperbolic Problems. Cambridge University Press, 2002.

6. R. J. LeVeque. Finite Difference Methods for Ordinary and Partial Differential Equations: Steady-State and Time-Dependent Problems. SIAM, Philadelphia, 2007.

7. R. L. LeVeque. Conservation law package (clawpack), 2003. Available at the URL http://depts.washington.edu/clawpack.

8. W. F. Noh and P. Woodward. SLIC (simple line interface calculation). In A. I. van de Vooren and P. J. Zandbergen, editors, Proc. 5th Intl. Conf. on Numer. Meth. in Fluid Dynamics. Springer-Verlag, 1976.

9. E. Olsson and G. Kreiss. A conservative level set method for two phase flow. J. Comput. Phys., 210:225-246, 2005.

10. E. Olsson, G. Kreiss, and S. Zahedi. A conservative level set method for two phase flow II. J. Comput. Phys., 225:785-807, 2007.

11. R. K. Shukla, C. Pantano, and J. B. Freund. An interface capturing method for the simulation of multi-phase compressible flows. J. Comput. Phys., 229:74117439, 2010.

12. K.-M. Shyue. A fluid-mixture type algorithm for compressible multicomponent flow with Mie-Grüneisen equation of state. J. Comput. Phys., 171:678-707, 2001.

13. K. K. So, X. Y. Hu, and N. A. Adams. Anti-diffusion method for interface steepening in two-phase incompressible flow. J. Comput. Phys., 230:5155-5177, 2011.

14. K. K. So, X. Y. Hu, and N. A. Adams. Anti-diffusion interface sharpening technique for two-phase compressible flow simulations. J. Comput. Phys., 231:43044323, 2012.

15. O. Ubbink and R. I. Issa. A method for capturing sharp fluid interfaces on arbitrary meshes. J. Comput. Phys., 153:26-50, 1999.

16. L. Štrubelj and I. Tiselj. Two-fluid model with interface sharpening. Int. J. Numer. Meth. Engng, 85:575-590, 2011.

17. F. Xiao, Y. Honma, and T. Kono. A simple algebraic interface capturing scheme using hyperbolic tangent function. Int. J. Numer. Mech. Fluids, 48:1023-1040, 2005. 